\title{
Canine Fetal Peritonitis
}

\section{Andreia Regis de Assis ${ }^{1,2}$, Marcus Antonio Rossi Feliciano ${ }^{3}$, Gilberto Gonçalves Facco ${ }^{4}$, Patricia Eri Ishii ${ }^{2}$, Cláudia Matsunaga Martin ${ }^{5} \&$ Carlos Eurico Fernandes ${ }^{2}$}

\begin{abstract}
Background: The occurrence of neonatal diseases and neonatal deaths in canines are considered higher in the first days of life of individuals and bacterial diseases are considered the second most common cause. Inflammation of the mesothelial lining of the abdominal cavity is referred peritonitis, which can be classified as primary or secondary and there is no description on the occurrence of canine fetal peritonitis in the veterinary literature. The objective of this study is to describe a case of canine fetus peritonitis, presenting abnormal sonographic findings of the fetus and abnormalities identified after birth that were not yet described in veterinary literature.

Case: A female Poodle, six years of age, considered healthy and without clinical complications during the gestational period, was referred for sonographic evaluation at the end of the pregnancy, in order to evaluate of the fetal viability and estimate the gestational period. By ultrasound examination, two canine fetuses were identified and based on its morphogenesis and biparietal diameter, the estimated gestational age was approximately 58 days. On the individual examination of each of the fetus by ultrasound, it was found that one of them had normal sonographic findings and on the second canine fetus it was found an increase of the intra-abdominal echogenicity of tissues, loss of the sonographic definition of the intestinal loops and presence of laminar anechoic areas between the interfaces of organs, consistent with abdominal effusion. Parturition occurred the following day of the ultrasound examination no complications and with the birth of two canine fetuses. About six hours after birth, one of the neonates showed dyspnea, hypothermia, areas of ecchymosis, suffusion in the ventral abdominal region, icteric and pale mucous membrane and the presence of abdominal fluid and came to death about 30 min after. Cytological analysis of the sample revealed exuberant amount of erythrocytes, fibrin and leukocytes. Culture revealed infection by Staphylococccus aureus and Enterococcus sp. Presence of bloody fluid in the abdominal cavity was observed at necropsy, with yellowish parietal surface of the thoracic and abdominal cavities and petechiae on the lungs, heart, stomach surfaces and intestinal mucous membrane. Microscopically, the lungs showed extensive areas of parenchymal consolidation, vascular congestion and intense inflammatory infiltrate in the alveolar wall; the liver was characterized by vascular congestion, areas of degeneration of hepatocytes and presence of calcified foci, necrosis and inflammatory infiltrates. Associating the results and findings of the exams was possible to conclude the diagnostic of fetal peritonitis. Discussion: The visualization of abnormal findings in affected fetus by a gestational ultrasound exam makes this report inedited and relevant for veterinary obstetrics. This demonstrates the importance of ultrasound exam in pregnant bitches for early detection of maternal fetal diseases. Presence of abdominal fluid and increased mesenteric ochogenicity associated with loss of the intestinal loop definition corroborates with the clinical suspicion of peritonitis in fetus. Findings on the anatomopathological examination, cytology and culture allowed conclusion of the cause of death. Bacterial forms in the cytological smear supported the diagnostic suspicion of peritonitis. Gestational ultrasound exam performed in this report was essential for proper conduct of the pregnant bitch and viability of the fetuses, suggesting that the detected fetal abnormalities showed predictive value in the viability of diseased individual. This case report contributes with the description of canine fetal peritonitis.
\end{abstract}

Keywords: canine, abnormal pregnancy, ultrasonography. 


\section{INTRODUCTION}

The occurrence of neonatal diseases and neonatal deaths in canines are considered higher in the first days of life of individuals due to alterations such as hypoxia, genetic abnormalities, teratogenesis, malformations, maternal disorders, immunological deficits, inadequate development of individuals, unfavorable environmental conditions and infectious agents. In this context, bacterial diseases are considered the second most common cause of neonatal diseases and deaths of these individuals [9].

Inflammation of the mesothelial lining of the abdominal cavity is referred peritonitis, which can be classified as primary or secondary. The primary condition is uncommon and it is considered a direct result of hematogenous infection and it coud be facilitated by deficient immunity of the patient [3]. There is no description on the occurrence of canine fetal peritonitis in the veterinary literature.

Gestational ultrasonography in female dogs is a diagnostic method considered accurate and innocuous for both bitches and their fetuses. It allows gestational diagnosis in order to estimate the gestational age and to identify fetal viability with possible abnormal conditions related to pregnancy, such as fetuses less developed in relation to gestational age, fetal resorption and abortion [6]. However there are few literature descriptions of abnormal sonograph findings in canine fetuses.

The objective of this study is to describe a case of canine fetus peritonitis, presenting abnormal sonographic findings of the fetus and abnormalities identified after birth that were not yet described in veterinary literature.

\section{CASE}

A female Poodle, 6 years of age, considered healthy and without clinical complications during the gestational period, was referred for sonographic evaluation at the end of the pregnancy, in order to evaluate of the fetal viability and estimate the gestational period.

Ultrasound examination was performed using ultrasound Mindray DP $6600^{1}$ with microconvex transducer multifrequency (5.0 to $8.0 \mathrm{MHz}$ ). Two canine fetuses were identified on the ultrasound exam and based on its morphogenesis and biparietal diameter, the estimated gestational age was approximately 58 days, according to literature [8, 10]. Moreover, according to the $\mathrm{M}$ mode, both fetuses had a mean heart rate of 220 beats per min (bpm).
On the individual examination of each of the fetus by ultrasound, it was found that one of them had normal sonographic findings, compatible with the estimated gestational age. However, on the second canine fetus it was found an increase of the intra-abdominal echogenicity of tissues, loss of the sonographic definition of the intestinal loops and presence of laminar anechoic areas between the interfaces of organs, consistent with abdominal effusion (Figure 1). The owner was suggested to return immediately to the responsible clinician of the case due to the changes identified in one of the fetuses.

The parturition occurred the following day of the ultrasound examination on the owner's property with no complications and with the birth of two canine fetuses. However, about $6 \mathrm{~h}$ after birth, one of the neonates showed dyspnea, hypothermia, areas of ecchymosis, suffusion in the ventral abdominal region (Figure 2), icteric and pale mucous membrane and the presence of fluid in the abdominal area. The puppy came to death about $30 \mathrm{~min}$ after ambulatory care, being forwarded to necropsy.

Prior to necropsy, paracentesis was performed with collection and shipment of abdominal fluid for laboratory evaluation and culture. The cytological analysis of the sample revealed exuberant amount of erythrocytes, fibrin and leukocytes represented by active macrophages, mixed lymphocytes, plasma cells, neutrophils and bacteria. The culture revealed infection by Staphylococccus aureus and Enterococcus sp.

Presence of bloody fluid in the abdominal cavity was observed at necropsy (Figure 2), with a yellowish parietal surface of the thoracic and abdominal cavities and petechiae on the lungs, heart, stomach surfaces and intestinal mucous membrane (Figure 2). It was not observed macroscopic evidence of congenital defects in the neonate. Through these findings suggestions were made that this was the fetus with abnormalities on the ultrasound exam.

Fragments of kidney, liver, heart, lung, brain, spleen, stomach, and intestines were collected and fixed in buffered formalin for inclusion in paraffin. Histopathological slides were prepared and stained with hematoxylin-eosin for evaluation under the optical microscope. Microscopically, the lungs showed extensive areas of parenchymal consolidation, vascular congestion and intense inflammatory infiltrate in the alveolar wall; the liver was characterized by vascular congestion, areas of degeneration of hepatocytes and presence of calcified 


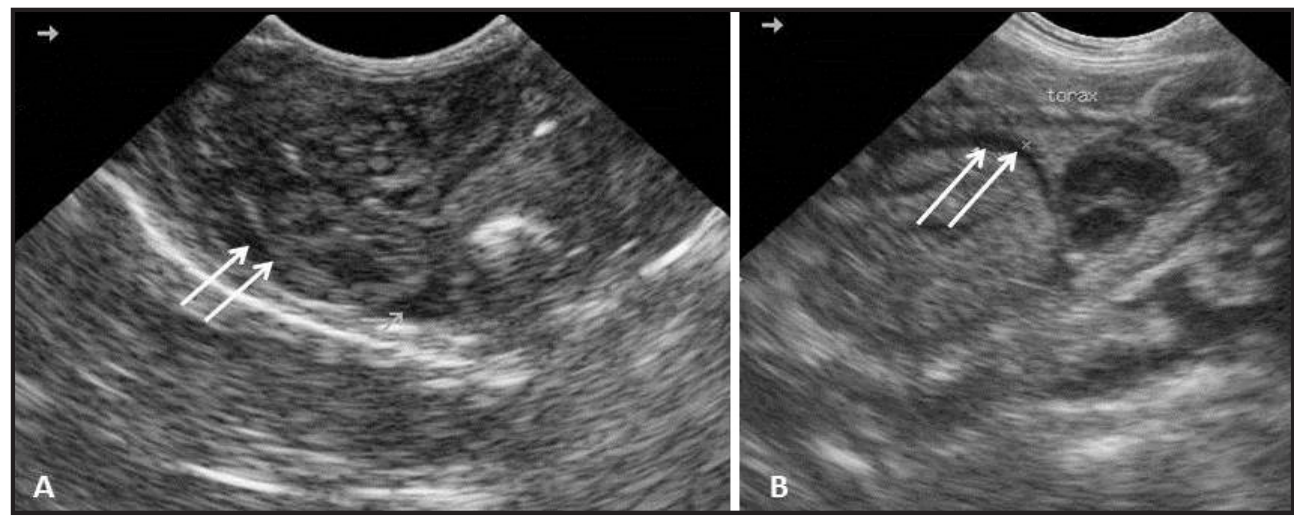

Figure 1. Sonographic Images of canine fetus with abdominal alterations. A- Loss of definition of the intestinal loops (arrows), interspersed with anechoic images; B- Presence of fluid (arrows) between liver and diaphragm region.
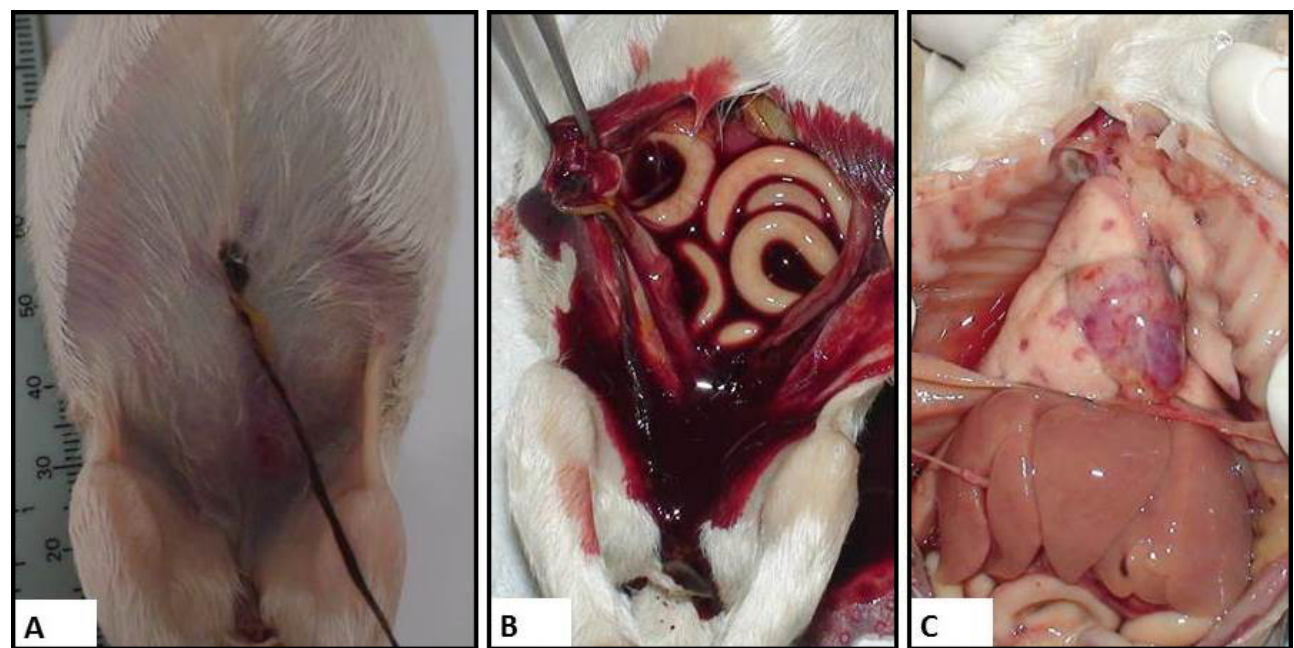

Figure 2. Photography of canine after death. A- Presence of ecchymosis in abdominal wall; B- Presence of exudate bloody in abundance and petechiae in lung tissue and abdominal organs (C).

foci, necrosis and inflammatory infiltrates. Other organs showed no microscopic alterations.

Associating the clinical symptoms and findings of the abdominal ultrasound exam, laboratory results and necropsy was possible to conclude that the death of the neonate was secondary to bacterial peritonitis, verified earlier by sonographic evaluation. A day after the parturition, the mother and the healthy puppy showed no clinical alteration and were both followed up during six months for clinical evaluations appearing to be healthy until this moment.

\section{DISCUSSION}

Peritonitis is a clinical abnormality of great importance in veterinary medicine, considered as one of the complications observed in newborns affected by perinatal bacterial infections [1]. The visualization of abnormal findings in the affected fetus by a gestational ultrasound exam makes this report inedited and relevant for veterinary obstetrics. This demonstrates the importance of ultrasound exam in pregnant bitches for early detection of maternal fetal diseases in this species [5].

According to the literature [8] on canine peritonitis cases, presence of abdominal fluid and increased mesenteric ochogenicity associated with loss of the intestinal loop definition corroborates with the clinical suspicion of peritonitis in the canine fetus.

Clinical findings observed on puppies with sepsis includes sudden death to severe septicemic disease, absence of suction, swallowing reflex changes, weight loss, dyspnea, hypothermia, diarrhea, abdominal discomfort, abdominal distention, weakness and stupor [9]. Some of these cited signs, although nonspecific, were observed in the diseased neonate and demonstrate the severity of the clinical condition observed.

Findings on the anatomopathological examination, cytology and abdominal fluid culture allowed conclusion of the neonate cause of death. Bacterial 
forms in the cytological smear supported the diagnostic suspicion of peritonitis. Microbiological culture confirmed the infection etiology with mixed pathogens as Staphylococcus aureus and Enterococcus sp., both present in canine neonatal infections [7].

Sonographic aspects of regular gestations are well established [2, 4], but the veterinary obstetrics needs to establish ultrasound protocols to evaluate the critical periods of the pregnant animal beyond the description of the possible abnormal findings of the major diseases in dogs and cats fetuses. In this context, gestational ultrasound exam performed in this report was essential for proper conduct of the pregnant bitch and viability of the fetuses, suggesting that the detected fetal abnormalities showed predictive value in the viability of diseased individual.

This case report contributes with the description of canine fetal peritonitis. Sonographic evaluation at the end of gestation in bitches can contribute to early assistance to both mother and neonate, favoring the establishment of adequate and effective therapy.

\section{MANUFACTURER}

${ }^{1}$ Mindray. Shenzhen, China.

Declaration of interest. The authors report no conflicts of interest. The authors alone are responsible for the content and writing of the paper.

\section{REFERENCES}

1 Blunden T. 2012. Fading puppies - reality or myth? In Practice. 34: 314-321.

2 Castro V.M., Mamprim M.J., Lopes M.D. \& Sartor R. 2011. Acompanhamento da gestação em cadelas pelo exame ultrassonográfico - revisão de literatura. Veterinaria \& Zootecnia. 18: 9-18.

3 D'Ávila G.F.L. 2012. Peritonite em cães. 48f. Monografia (Graduação em Medicina Veterinária) - Universidade Federal do Rio Grande do Sul, Faculdade de Veterinária, Porto Alegre.

4 Feliciano M.A.R., Muzzi L.A.L., Leite C.A.L. \& Junqueira M.A. 2007. Two-dimensional conventional, high resolution two-dimensional and three-dimensional ultrasonography in the evaluation of pregnant bitch. Arquivo Brasileiro de Medicina Veterinária \& Zootecnia. 59: 1333-1337.

5 Feliciano M.A.R., Cardilli D.J., Crivelaro R.M., Garrido E., Silva M.A.M., Castanheira T.L.L. \& Vicente W.R.R. 2013. Hydrallantois in a female dog: a case report. Arquivo Brasileiro de Medicina Veterinária \& Zootecnia. 65: 10911095.

6 Feliciano M.A.R., Maciel G.S., Coutinho L.N., Almeida V.T., Uscategui R.R. \& Vicente W.R.R. 2015. Gestational echo biometry in brachycephalic pregnant bitches. Ciência Animal Brasileira. 16: 419-427.

7 Löhr C.V. 2011. Exame post Mortem dos filhotes de cães e gatos. In: Peterson M.E. \& Kuztler M.A. (Eds). Pediatria em pequenos animais. Rio de Janeiro: Elsevier, pp.276-287.

8 Mattoon T.G. \& Nyland J.S. 2015. Small Animal Diagnostic Ultrasound. 3rd edn. São Paulo: Roca, 667p.

9 Münnich A. \& Küchenmeister U. 2014. Causes, Diagnosis and therapy of common diseases in neonatal puppies in the first days of life: cornerstones of practical approach. Reproduction in Domestic Animals. 49: 64-74.

10 Socha P., Rudowska M. \& Janowski T. 2012. Effectiveness of determining the parturition date in bitches using the ultrasonographic fetometry as compared to hormonal and cytological methods. Polish Journal of Veterinary Sciences. 15: 447-453. 